\title{
Oxone-Mediated Preparation of Ester Derivatives Using Indium(III) Triflate and Various Alcohols
}

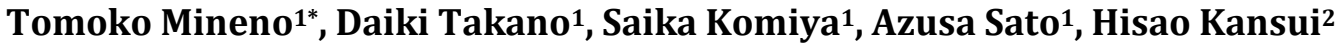 \\ ${ }^{1}$ Laboratory of Medicinal Chemistry, Faculty of Pharmacy, Takasaki University of Health and Welfare, Takasaki, Gunma, Japan \\ ${ }^{2}$ Laboratory of Organic Chemistry, Faculty of Pharmaceutical Sciences, Sojo University, Ikeda, Kumamoto, Japan \\ Email: *mineno@takasaki-u.ac.jp
}

How to cite this paper: Mineno, T., Takano, D., Komiya, S., Sato, A. and Kansui, H. (2022) Oxone-Mediated Preparation of Ester Derivatives Using Indium(III) Triflate and Various Alcohols. Green and Sustainable Chemistry, 12, 1-8.

https://doi.org/10.4236/gsc.2022.121001

Received: January 6, 2022

Accepted: February 14, 2022

Published: February 17, 2022

Copyright (C) 2022 by author(s) and Scientific Research Publishing Inc. This work is licensed under the Creative Commons Attribution International License (CC BY 4.0).

http://creativecommons.org/licenses/by/4.0/

\begin{abstract}
Esters are known as one of the most fundamental chemical moieties and also essentially useful components, especially for medicinal agents. Herein, using benzaldehyde and its derivatives as starting materials, Oxone-mediated preparation of ester derivatives in the presence of a catalytic amount of indium(III) triflate is described. Alcohols with various chain lengths, which functioned as solvents and substrates, were examined. Overall, the oxidative esterification starting with benzaldehyde derivatives possessing electron withdrawing groups proceeded smoothly and gave sufficient yields, in comparison to the reactions with the derivatives having electron donating groups.
\end{abstract}

\section{Keywords}

Oxone, Oxidative Esterification, Benzaldehyde Derivatives, Ester Derivatives, Indium(III) Triflate, Alcohols

\section{Introduction}

Esters are popular as one of the most fundamental chemical moieties, being composed of a combination of carboxylic acids and alcohols. Among esters, methyl esters are often primarily utilized for protecting the functionality of carboxylic acids because leaving carboxylic acids in their unprotected free forms could cause unwanted side reactions upon implementing certain chemical reactions. Meanwhile, in the fields of medication and chemotherapy, a dose of medicines that possesses ester moieties can often serve as prodrugs with improved bioavailability. Due to enhanced lipophilicity by esterification on a parent drug, improved pharmacological properties such as increased oral and transdermal 
absorption would be expected. Esters are generally subjected to hydrolysis in their active form in the body by way of the function of esterase. As an example of esters, osertamivir, one of the anti-influenza agents, has the structure of ethyl ester to raise oral bioavailability, which is followed by cleavage into the active form of a carboxylate [1]. Also, dexamethasone 17-valerate and dexamethasone 17,21-dipropionate are formulated agents in dermatological medication to treat skin inflammation, for example. Their topical efficacy is promoted through the form of valerate and propionate esters respectively that enhance transdermal absorption [2] [3].

We have been conducting research on indium and indium related reagents and have reported an efficient method of high-yielding methyl esterification, which utilizes trivalent $\mathrm{InCl}_{3}$ under mild reaction conditions [4]. Other examples of the preparation of methyl esters include a reaction by stirring carboxylic acids in methanol in the presence of a catalytic amount of trimethylsilyl chloride [5]. Throughout our continuous study, we have developed Oxone-mediated oxidative methyl esterification of benzaldehyde derivatives [6]. The reactions were accelerated in the presence of $\operatorname{In}(\mathrm{OTf})_{3}$, via the use of an effective oxidant, Oxone monopersulfate compound (Oxone), which is composed of a triple salt of potassium peroxymonosulfate. Oxone is a well-known stable oxidant with versatile features that enable a wide variety of applications [7]. Oxone, as an example, can be an efficient oxidant for the epoxidation of alkenes [8] [9] [10] [11] [12]. In another example, Oxone can function for the conversion from sulfides to sulfones [13] [14]. The use of Oxone with wet-Alumina processes the ring expansion from cyclic ketones to lactones [15], and this kind of ring expansion can also be carried out by using Oxone in the presence of ionic liquids as solvents through the way of Beayer-Villiger oxidation [16]. We then applied Oxonemediated reactions to heterocyclic aldehydes with the usage of methanol as well as other alcohols having various chain lengths [17]. Ester derivatives are often prepared to increase therapeutic effects of drugs through improved bioavailability. Enhanced lipophilicity of ester derivatives can be indicated to resolve some difficulties in therapeutic procedures. This research now will become a desirable solution to upgrade public health. In the view of these previously reported esterification methodologies [6] [17] [18], we thereafter further explored Oxonemediated preparation of esters on benzaldehyde and its derivatives as starting materials in the presence of a catalytic amount of $\operatorname{In}(\mathrm{OTf})_{3}$ and investigated alcohols with various chain lengths, which function as solvents and substrates. The details of our studies are presented herein.

\section{Results and Discussion}

First, we initiated examination of Oxone-mediated preparation of ester derivatives using benzaldehyde as starting material as in Table 1. Applying the reaction condition from our previous work [6] [17], oxidative esterification was carried out in alcohols with elongated carbon chains. The desired products of methyl 
Table 1. Reactions using benzaldehyde and various alcohols.

Entry ${ }^{\mathrm{a}}$

${ }^{a}$ All reactions were carried out in the presence of 1.0 equiv. of Oxone and $10 \mathrm{~mol} \%$ of

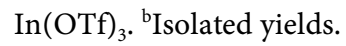

ester [19] [20], ethyl ester [21], propyl ester [22], and butyl ester [23] were furnished respectively without any experimental difficulties (Table 1, entries 1 - 4). Upon comparing the yields, a gradual increase is observed from $63 \%$ in methanol in entry 1 to $86 \%$ in butanol in entry 4 , which can be occurred in accordance with boiling point raising from methanol to butanol at reflux. However, in the case of heavier alcohols such as pentanol and hexanol, the reactions did not proceed properly, instead, they turned the reaction mixtures into blackened solutions. Perhaps, physical properties, like viscosity and less polarity of these heavier alcohols, can be the cause of these unproductive results.

Having desired yields of benzaldehyde, we next conducted Oxone-mediated reactions starting with benzaldehyde derivatives possessing either electron donating groups or electron withdrawing groups at the C-4 position on the benzene ring. In the case of 4-tolualdehyde in methanol, a good yield of $82 \%$ has been reported previously (Table 2, entry 1) [6]. However, further attempts in ethanol, propanol, and butanol gave unsatisfactory yields (Table 2, entries 2 - 4) [23] [24] [25], prompting us to carry out the identical reactions starting with 4 -anisaldehyde derivatives that has a methoxy group at the C-4 position. The outcome was similar, the reaction in methanol furnished the product in a good yield of $82 \%$ (Table 2, entry 5), but the reactions in ethanol, propanol, and butanol yielded them in less than $10 \%$ (Table 2, entries 6 - 8) [23] [24]. Consequently, we further expanded our exploration to benzaldehyde derivatives with electron withdrawing groups at the C-4 position. In the case of 4-chlorobenzaldehyde, although the reaction in methanol gave a moderate yield of $73 \%$ in $3 \mathrm{~h}$ 
Table 2. Reactions using benzaldehyde with electron donating groups and various alcohols.

Entry

${ }^{a}$ All reactions were carried out in the presence of 1.0 equiv. of Oxone and $10 \mathrm{~mol} \%$ of

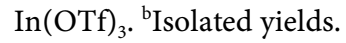

(Table 3, entry 1), the reactions in ethanol, propanol, and butanol afforded the corresponding products in more than $80 \%$ yields in slightly longer reaction time (Table 3, entries 2 - 4) [23] [24] [26]. The reactions starting with 4-cyanobenzaldehyde also proceeded smoothly (Table 3, entries 5 - 8) [27], including excellent yields of $94 \%$ in methanol (Table 3 , entry 5). Furthermore, the oxidative esterification with 4-nitorobenzaldehyde, which has a very strong electron withdrawing group at the C-4 position, were examined, and it unexpectedly turned out that the reactions in longer carbon chains of propanol and butanol displayed higher productivity of $88 \%$ and $96 \%$ yields (Table 3, entries 11 and 12) [28] [29] than the yields of $70 \%$ and $35 \%$ through the reactions in methanol and ethanol (Table 3, entries 9 and 10) [27]. 
Table 3. Reactions using benzaldehyde with electron withdrawing groups and various alcohols.

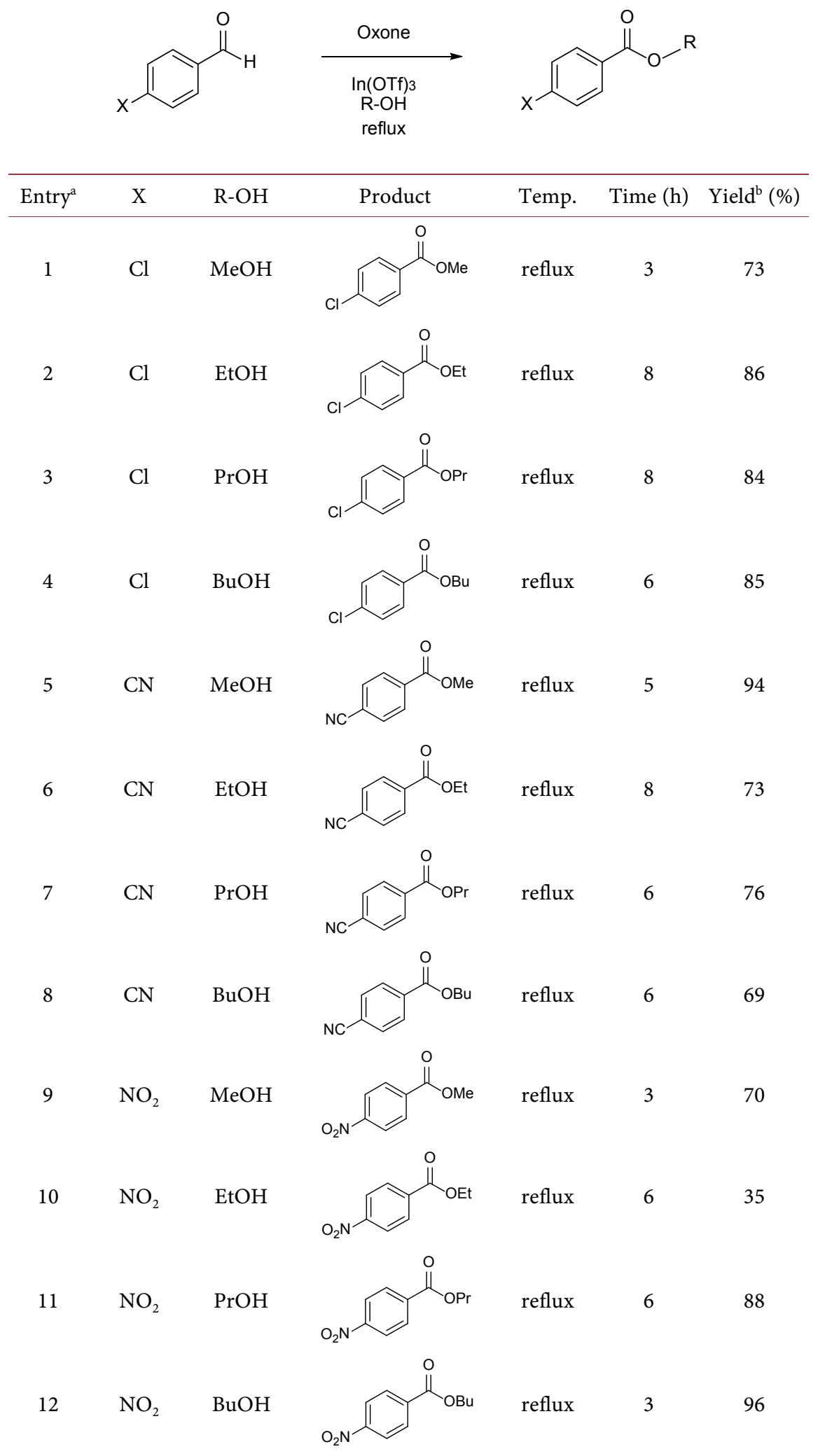

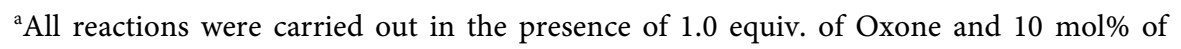

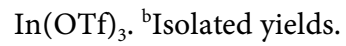




\section{Conclusion}

In conclusion, we have investigated Oxone-mediated preparation of ester derivatives in the presence of a catalytic amount of $\operatorname{In}(\mathrm{OTf})_{3}$. As starting materials, benzaldehyde and its derivatives were subjected to the reaction conditions with various alcohols. Comparing benzaldehyde derivatives with electron donating groups and ones with electron withdrawing groups at the C-4 position, the reactions overall went on smoothly and produced corresponding esters in higher yields, in the case of benzaldehyde derivatives with electron withdrawing groups than the derivatives with electron donating groups, noticeably upon implementation in alcohols with longer carbon chains.

\section{Experimental}

\subsection{Materials and Instruments}

All reagents were of analytical grade purchased commercially and used without further purification. All reactions were performed under argon using magnetic stirring unless otherwise stated. ${ }^{1} \mathrm{H}$ NMR and ${ }^{13} \mathrm{C}$ NMR spectral data were recorded on a JEOL JMTC-500 spectrometer using TMS as the internal standard.

\subsection{General Experimental Procedure}

Benzaldehyde ( $5 \mathrm{mmol}$ ) as starting material was combined with Oxone ( $5 \mathrm{mmol}$ ) and $\mathrm{In}(\mathrm{OTf})_{3}(10 \mathrm{~mol} \%)$ in methanol $(50 \mathrm{~mL})$. The reaction mixture was refluxed and monitored for completion by TLC. The reaction mixture was filtered, and the filtrate was condensed by rotary evaporation. The resulting residue was purified by silica gel flash column chromatography to obtain the desired esters, which were confirmed by spectroscopy [6] [19]-[29].

4-Methoxybenzoic acid propyl ester: ${ }^{1} \mathrm{H}$ NMR (500 MHz, Chloroform- $d$ ) $\delta$ $8.00(\mathrm{~d}, 2 \mathrm{H}, J=9.2 \mathrm{~Hz}), 6.91(\mathrm{~d}, 2 \mathrm{H}, J=9.2 \mathrm{~Hz}), 4.24(\mathrm{t}, 2 \mathrm{H}, J=6.9 \mathrm{~Hz}), 3.85(\mathrm{~s}$, $3 \mathrm{H}), 1.77$ (sext, 2H, $J=7.5 \mathrm{~Hz}), 1.02(\mathrm{t}, 3 \mathrm{H}, J=7.5 \mathrm{~Hz}) ;{ }^{13} \mathrm{C}$ NMR $(125 \mathrm{MHz}$, Chloroform-d) $\delta 166.4,163.2,131.5,122.9,113.5,66.2,55.3,22.1,10.5$.

4-Cyanobenzoic acid propyl ester: ${ }^{1} \mathrm{H}$ NMR $(500 \mathrm{MHz}$, Chloroform- $d$ ) $\delta$ $7.99(\mathrm{~d}, 2 \mathrm{H}, J=8.6 \mathrm{~Hz}), 7.61(\mathrm{~d}, 2 \mathrm{H}, J=8.6 \mathrm{~Hz}), 4.17(\mathrm{t}, 2 \mathrm{H}, J=6.3 \mathrm{~Hz}), 1.66$ (sext, $2 \mathrm{H}, J=7.5 \mathrm{~Hz}), 0.89(\mathrm{t}, 3 \mathrm{H}, J=7.45 \mathrm{~Hz}) ;{ }^{13} \mathrm{C}$ NMR $(125 \mathrm{MHz}$, Chloroform-d) $\delta 164.3,133.8,131.7,129.5,117.4,115.7,66.7,21.5,9.9$.

4-Cyanobenzoic acid butyl ester: ${ }^{1} \mathrm{H}$ NMR $(500 \mathrm{MHz}$, Chloroform- $d$ ) $\delta 8.11$ $(\mathrm{d}, 2 \mathrm{H}, J=8.6 \mathrm{~Hz}), 7.71(\mathrm{~d}, 2 \mathrm{H}, J=8.6 \mathrm{~Hz}), 4.33(\mathrm{t}, 2 \mathrm{H}, J=6.6 \mathrm{~Hz}), 1.73$ (quint, $2 \mathrm{H}, J=7.5 \mathrm{~Hz}$ ), 1.45 (sext, $2 \mathrm{H}, J=7.5 \mathrm{~Hz}), 0.95$ (t, 3H, $J=7.5 \mathrm{~Hz}$ ); ${ }^{13} \mathrm{C}$ NMR (125 MHz, Chloroform-d) $\delta 164.8,134.2,132.1,129.9,117.9,116.1,65.5,30.5$, 19.1, 13.6.

\section{Conflicts of Interest}

The authors declare no conflicts of interest regarding the publication of this report. 


\section{References}

[1] Chang, Q., Chow, M.S.S. and Zuo, Z. (2009) Studies on the Influence of Esterase Inhibitor to the Pharmacokinetic Profiles of Oseltamivir and Oseltamivir Carboxylate in Rats Using an Improved LC/MS/MS Method. Biomedical Chromatography, 23, 852-857. https://doi.org/10.1002/bmc.1195

[2] Fregnan, G.B. and Torsello, A.L. (1975) Topical Anti-Inflammatory Activity of Dexamethasone 17-Valerate and Other Corticosteroids. Current Therapeutic Researc, 17, 375-381.

[3] Fujiwara, K., Ajioka, H. and Miyake, H. (1984) Anti-Inflammatory Effect of Dexamethasone 17,21-Dipropionate (THS-101) (2). Oyo Yakuri-Pharmacometrics, 28, 653661.

[4] Mineno, T. and Kansui, H. (2006) High Yielding Methyl Esterification Catalyzed by Indium(III) Chloride. Chemical \& Pharmaceutical Bulletin, 54, 918-919. https://doi.org/10.1248/cpb.54.918

[5] Mineno, T. and Miller, M.J. (2003) Stereoselective Total Synthesis of Racemic BCX1812 (RWJ-270201) for the Development of Neuraminidase Inhibitors as AntiInfluenza Agents. The Journal of Organic Chemistry, 68, 6591-6596. https://doi.org/10.1021/jo034316b

[6] Mineno, T., Sakai, M., Ubukata, A., Nakahara, K., Yoshimitsu, H. and Kansui, H. (2013) The Effect of Indium Triflate(III) in Oxone-Mediated Oxidative Methyl Esterification of Aldehydes. Chemical \& Pharmaceutical Bulletin, 61, 870-872. https://doi.org/10.1248/cpb.c13-00072

[7] Marcotullio, M.C., Epifano, F. and Curini, M. (2003) Recent Advances in the Use of Oxone ${ }^{\circledast}$ in Organic Synthesis. Trends in Organic Chemistry, 10, 21-34.

[8] Hussain, H., Green, I.R. and Ahmed, I. (2013) Journey Describing Applications of Oxone in Synthetic Chemistry. Chemical Reviews, 113, 3329-3371.

https://doi.org/10.1021/cr3004373

[9] Armstrong, A., Ahmed, G., Garnett, I. and Goacolou, K. (1997) Pyrrolidine-Derived Iminium Salts as Catalysts for Alkene Epoxidation by Oxone. Synlett, No. 9, 1075 1076. https://doi.org/10.1055/s-1997-1542

[10] Denmark, S.E., Wu, Z., Crudden, C.M. and Matsuhashi, H. (1997) Catalytic Epoxidation of Alkenes with Oxone, 2. Fluoro Ketones. The Journal of Organic Chemistry, 62, 8288-8289. https://doi.org/10.1021/jo971781y

[11] Hashimoto, N. and Kanda, A. (2002) Practical and Environmentally Friendly Epoxidation of Olefins Using Oxone. Organic Process Research \& Development, 6, 405-406. https://doi.org/10.1021/op025511f

[12] Armstrong, A. (2004) Amine-Catalyzed Epoxidation of Alkenes: A New Mechanism for the Activation of Oxone. Angewandte Chemie International Edition, 43, 1460 1462. https://doi.org/10.1002/anie.200301716

[13] Trost, B.M. and Curran, D.P. (1981) Chemoselective Oxidation of Sulfides to Sulfones. Tetrahedron Letters, 22, 1287-1290. https://doi.org/10.1016/S0040-4039(01)90298-9

[14] Mahajan, N.S., Jadhav, R.L., Pimpodkar, N.V., Dias, R.J. and Manikrao, A.M. (2009) Green Solid Oxidation of Silfides to Sulfones Using Oxone and Biological Evaluation. Asian Journal of Chemistry, 21, 5415-5420.

[15] Hirano, M., Oose, M. and Morimoto, T. (1991) A Novel Synthesis of Lactones by the Oxidation of Alicyclic Ketones with Oxone in Aprotic Solvent in the Pressure of Wet-Alumina. Chemistry Letters, 331-332. https://doi.org/10.1246/cl.1991.331 
[16] Chrobok, A. (2010) The Baeyer-Villiger Oxidation of Ketones with Oxone ${ }^{\circledast}$ in the Presence of Ionic Liquids as Solvents. Tetrahedron, 66, 6212-6216. https://doi.org/10.1016/j.tet.2010.05.091

[17] Mineno, T., Yoshino, S. and Ubukata, A. (2014) Oxone-Mediated Oxidative Esterification of Heterocyclic Aldehydes Using Indium(III) Triflate. Green and Sustainable Chemistry, 4, 20-23. https://doi.org/10.4236/gsc.2014.41004

[18] Travis, B.R., Silvakumar, M., Hollist, G.O. and Borhan, B. (2003) Facile Oxidation of Aldehydes to Acids and Esters with Oxone. Organic Letters, 5, 1031-1034. https://doi.org/10.1021/ol0340078

[19] Abraham, R.J., Bardsley, B., Mobli, M. and Smith, R.J. (2005) ${ }^{1} \mathrm{H}$ Chemical Shifts in NMR. Part $21^{+}$-Prediction of the ${ }^{1} \mathrm{H}$ Chemical Shifts of Molecules Containing the Ester Group: A Modelling and ab Initio Investigation. Magnetic Resonance in Chemistry, 43, 3-15. https://doi.org/10.1002/mrc.1491

[20] Sequin, U. (1981) Carbon-13 NMR Spectral Differences between Corresponding Methyl Esters, Phenyl Esters and 2-Substituted Chromones. Helvetica Chimica Acta, 64, 2654-2664. https://doi.org/10.1002/hlca.19810640824

[21] Schmidt, A., Habeck, T., Snovydovych, B. and Eisfeld, W. (2007) Addition Reactions and Redox Esterifications of Carbonyl Compounds by N-Heterocyclic Carbenes of Indazole. Organic Letters, 9, 3515-3518. https://doi.org/10.1021/ol0713739

[22] Gowrisankar, S., Neumann, H. and Beller, M. (2011) General and Selective Palladium-Catalyzed Oxidative Esterification of Alcohols. Angewandte Chemie International Edition, 50, 5139-5143. https://doi.org/10.1002/anie.201008035

[23] Liu, Q., Li, G., He, J., Liu, J., Li, P. and Lei, A. (2010) Palladium-Catalyzed Aerobic Oxidative Carbonylation of Arylboronate Esters under Mild Conditions. Angewandte Chemie International Edition, 49, 3371-3374. https://doi.org/10.1002/anie.201000460

[24] Hu, Y., Liu, J., Lu, Z., Luo, X., Zhang, H., Lan, Y. and Lei, A. (2010) Base-Induced Mechanistic Variation in Palladium-Catalyzed Carbonylation of Aryl Iodides. Journal of the American Chemical Society, 132, 3153-3158.

https://doi.org/10.1021/ja909962f

[25] Mercadante, M.A. and Leadbeater, N.E. (2011) Continuous-Flow, PalladiumCatalysed Alkoxycarbonylation Reactions Using a Prototype Reactor in Which It Is Possible to Load Gas and Heat Simultaneously. Organic and Biomolecular Chemistry, 9, 6575-6578. https://doi.org/10.1039/c1ob05808h

[26] Yamamoto, Y. (2010) The First General and selective Palladium(II)-Catalyzed Alkoxycarbonylation of Arylboronates: Interplay among Benzoquinone-Ligated Palladium(0) Complex, Organoboron, and Alcohol Solvent. Advanced Synthesis \& Catalysis, 352, 478-492. https://doi.org/10.1002/adsc.200900836

[27] Shang, R., Fu, Y., Li, J.-B., Zhang, S.-L., Guo, Q.-X. and Liu, L. (2009) Synthesis of Aromatic Esters via Pd-Catalyzed Decarboxylative Coupling of Potassium Oxalate Monoesters with Aryl Bromides and Chlorides. Journal of the American Chemical Society, 131, 5738-5739. https://doi.org/10.1021/ja900984x

[28] Kiran, Y.B., Ikeda, R., Sakai, N. and Konakahara, T. (2010) Single-Step Conversion of Electron-Deficient Aldehydes into the Corresponding Esters in Aqueous Alcohols in the Presence of Iodine and Sodium Nitrite. Synthesis, No. 2, 276-282. https://doi.org/10.1055/s-0029-1217121

[29] Hashmi, A.S.K., Lothschuetz, C., Ackermann, M., Doepp, R., Anantharaman, S., Marchetti, B., Bertagnolli, H. and Rominger, F. (2010) Gold Catalysis: In Situ EXAFS Study of Homogeneous Oxidative Esterification. Chemistry. A European Journal, 16, 8012-8019. https://doi.org/10.1002/chem.200903450 